\title{
Programa cognitivo conductual para fortalecer habilidades de comunicación enfermera paciente
}

\section{Cognitive-behavioral program to strengthen the nurse-patient communication}

\section{Programa cognitivo comportamental para fortalecer habilidades de comunicação enfermeira paciente}

\author{
M.C. Müggenburg-RodríguezVigil ${ }^{a 1^{*}}$, M.C. Hernández Guillén ${ }^{2}$, A. Aldana ${ }^{2}$ \\ ORCID: \\ a $0000-0002-0497-8685$ \\ ${ }^{1}$ Unidad de Investigación, Escuela Nacional de Enfermería y Obstetricia, Universidad Nacional Autónoma de \\ México, Ciudad de México, México \\ ${ }^{2}$ División de Estudios Profesionales, Escuela Nacional de Enfermería y Obstetricia, Universidad Nacional Autó- \\ noma de México, Ciudad de México, México
}

Recibido: 14 noviembre 2017

Aceptado: 8 octubre 2018

\section{Resumen}

Introducción: La comunicación enfermera paciente es eje articulador de los cuidados, requiere habilidades específicas de comunicación verbal y no verbal. El entrenamiento con estrategias cognitivo conductuales para el fomento de estas habilidades coadyuva en forma importante en el establecimiento de relaciones terapéuticas de la práctica profesional de enfermería; estas técnicas han sido ampliamente aplicadas, en el presente caso se utilizó el Aprendizaje Estructurado, cuyo objetivo se centra en el cambio de comportamiento, sin dejar de reconocer que también propicia cambios favorables en sentimientos y actitudes. Este artículo se enfoca en dar a conocer la aplicación práctica de un programa para enfermeras sobre el fortalecimiento de habilidades de comunicación.

Desarrollo: Las habilidades seleccionadas en el desarrollo de este programa con el apoyo de la estrategia Aprendizaje Estructurado fueron: Escuchar, Expresar aliento, Expresar un cumplido, Responder a los sentimientos del otro, Responder a una queja, Responder al enojo y Expresar afecto.

Se registraron conductas verbales y no verbales individuales durante el entrenamiento, para mostrar la presencia o ausencia del comportamiento, en su caso la adquisición y el número de ensayos necesarios para lograrlo. Se presentan los registros de tres de las siete habilidades incluidas en el programa, con la finalidad de dar a conocer el desarrollo del aprendizaje durante la implementación. 
Conclusiones: El programa de entrenamiento dio la oportunidad de fortalecer habilidades de comunicación en las enfermeras, especialmente en el ámbito de la comunicación verbal, elemento que favorecerá una mayor identificación entre enfermeras y pacientes con el indudable beneficio para la detección de necesidades del paciente y establecimiento de planes de cuidado.

Palabras clave: Comunicación; relación enfermero-paciente; investigación en enfermería; México.

\section{Abstract}

Introduction: Nurse-patient communication is the axis of care provision, and thus requires specific verbal and non-verbal abilities. Training using cognitive behavioral strategies can help establish the appropriate therapeutic relationships of the nursing professional practice. These methodologies have been broadly utilized, and we used the Structured Learning approach which promotes behavior changes, including improvements in the feelings and attitudes. This article focuses on publicizing the practical application of a program for nurses on strengthening communication skills.

Development: The skills of interest were: Listening; Expressing value; Expressing compliment; Responding to the feelings of others; Responding to a complaint; Responding to anger; and Expressing affection.

Individual verbal and non-verbal behaviors were registered during the training sessions to acknowledge the absence of the desired behaviors, as well as number of trials needed to acquire the abilities of interest. In order to show the development of the program, registers of three of the seven abilities considered are displayed.

Conclusions: The training program provided the opportunity to strengthen the communication abilities of nurses, particularly around verbal communication. The resulting improvements will favor a greater identification and interaction between nurses and patients and will allow the design and execution of better plans of care.

Keywords: Communication; nurse-patient relationship; nursing research; Mexico.

\section{Resumo}

Introdução: A comunicação enfermeira-paciente é eixo articulador dos cuidados, requer habilidades específicas de comunicação verbal e não verbal. O treinamento com estratégias cognitivo-comportamentais para o fomento destas habilidades contribui em forma importante no estabelecimento de relações terapêuticas da prática profissional de enfermagem; estas técnicas foram largamente aplicadas, no presente caso utilizou-se a Aprendizagem Estruturada, cujo objetivo centra-se na mudança de comportamento, sem deixar de reconhecer que também propicia câmbios favoráveis em sentimentos e atitudes.

Deste artigo focaliza-se em dar a conhecer a aplicação prática de um programa para enfermeiras sobre o fortalecimento de habilidades de comunicação.

Desenvolvimento: As habilidades escolhidas no desenvolvimento deste programa com o apoio da estratégia Aprendizagem Estruturada foram: Escutar, Encorajar, Expressar um elogio, Responder aos sentimentos do outro, Responder a uma queixa, Responder à raiva e Expressar afeto.

Realizaram-se registros de comportamentos verbais e não verbais individuais durante o treinamento, com o fim de mostrar a presença ou ausência do comportamento, em seu caso a aquisição e o número de ensaios necessários para consegui-lo. Apresentaram-se os registros de três das sete habilidades incluídas no programa, com a finalidade de dar a conhecer o desenvolvimento da aprendizagem durante a implementação.

Conclusões: A execução do programa de treinamento deu a oportunidade de fortalecer habilidades de comunicação nas enfermeiras, especialmente no âmbito da comunicação verbal, elemento que favorecerá uma maior identificação entre enfermeiras e pacientes com o indubitável benefício para a detecção de necessidades do paciente e estabelecimento de planos de cuidado.

Palavras chave: Comunicação; relação enfermeiro-paciente; pesquisa em enfermagem; México. 


\section{Introducción}

La comunicación que se establece en el ejercicio de la práctica profesional de las enfermeras con sus pacientes, se realiza en tres fases: inicial, implica presentación, disponibilidad de tiempo y clarificación de la demanda del paciente; intermedia, conlleva la obtención de datos, la comprensión y el logro de la empatía; y la fase final, corresponde al cierre temporal de la relación ${ }^{1,2}$.

La comunicación interpersonal tiene dos formas de expresión: comunicación verbal y comunicación no verbal, en el caso de la enfermería va dirigida a la búsqueda de una relación terapéutica que incorpora ambas formas de comunicación, fundamentales al brindar el cuidado profesional, tanto las expresiones verbales como las corporales, la palabra idónea y su gesto congruente ${ }^{3-5}$.

El entrenamiento en habilidades de comunicación en enfermería no es nuevo, se ha realizado, se realiza y se seguirá realizando por la importancia que la comunicación ocupa como eje articulador de los cuidados. Las estrategias para los entrenamientos han sido variadas, muchas de ellas orientadas a técnicas cognitivo conductuales, sin embargo, los entrenamientos realizados y referidos ${ }^{6-11}$, no han agotado esta estrategia de formación en las instituciones de salud, por este motivo, en este espacio se presenta el análisis de la experiencia de formación con la aplicación de un programa de entrenamiento de las enfermeras en habilidades de comunicación con el paciente que recibe su cuidado ${ }^{11}$.

El aprendizaje estructurado es un método activo para adquirir nuevas habilidades que conducen a cambios positivos en las actitudes del principiante (confianza en sí mismo, respeto propio, sentido del valor personal o de las aptitudes propias). El objetivo directo es el cambio de comportamiento, pero también se esperan cambios favorables en los sentimientos y actitudes del aprendiz ${ }^{12}$; para aprender bien algo es necesario ser activo, el observar o leer enseña qué es lo que hay que hacer, pero el practicar o ensayar una habilidad enseña más plenamente el cómo hacerlo. Se necesitan ambas para un aprendizaje efectivo y duradero.

El entrenamiento se llevó a cabo con 14 enfermeras distribuidas en cuatro subgrupos, que laboraban en un servicio de hospitalización de una institución de salud de la ciudad de México de carácter público, durante mes y medio con sesiones de una hora semanal, se aplicó la estrategia cognitivo conductual denominada aprendizaje estructurado ${ }^{12,13}$. El entrenamiento fue impartido por una enfermera psicóloga con experiencia en técnicas cognitivo conductuales y dos facilitadoras entrenadas.

Se modelaron y practicaron habilidades sociales orientadas al desarrollo de condiciones facilitadoras de la relación terapéutica: empatía y respeto ${ }^{3}$. Se efectuaron registros individuales por enfermera, que incluyen criterios derivados de los componentes verbales y no verbales de cada habilidad, con el fin de retroalimentar a cada participante el número de veces necesario para alcanzar la habilidad.

El objetivo es dar a conocer la aplicación práctica de un programa de intervención para enfermeras relacionado con el fortalecimiento de habilidades de comunicación, además de considerar la importancia de éstas para el ejercicio de la práctica profesional en enfermería, dado que a mayor comunicación se logrará mayor adecuación entre las necesidades biológicas, psicológicas, sociales y espirituales y los cuidados brindados por las enfermeras, cuidados que pasarán a ser individualizados con el beneficio que esto conlleva.

\section{Desarrollo}

De acuerdo con la estrategia seleccionada para la aplicación del programa educativo los pasos empleados fueron: el diagnóstico inicial de cada enfermera respecto a la habilidad en cuestión, seguido del modelamiento de dicha habilidad por dos de las facilitadoras, después cada enfermera realizó un ensayo conductual, seguido de la retroalimentación, que permitió volver a ejecutar el ensayo, hasta que de acuerdo con las observaciones de dos de las facilitadoras, la enfermera mostraba los componentes verbales y no verbales que integran la habilidad, al finalizar cada sesión se les entregó un formato con los componentes de la habilidad practicada, con el propósito de que los ejercitaran en la semana, tanto con los pacientes como con alguna 
persona con la que tuvieran interacción personal habitualmente, para propiciar la transferencia de la habilidad a su vida diaria.

Se seleccionaron siete habilidades de un total de 48 , descritas por Gershaw et al. ${ }^{12}$, quiénes abordan de forma práctica el Aprendizaje Estructurado. La elección estuvo sustentada en los componentes que resultaban útiles para favorecer la comprensión empática y el respeto en la relación interpersonal enfermera paciente; Escuchar, Expresar aliento, Expresar un cumplido, Responder a los sentimientos de otro, Responder a una queja, Responder al enojo y Expresar afecto.

Cada una de estas siete habilidades quedó plasmada en un formato para registro individual que mostraba componentes verbales y componentes no verbales, empleados como criterios de adquisición de la habilidad, mismos que fueron observados y registrados por dos de las facilitadoras del programa. Conforme el esquema del Aprendizaje Estructurado se realizó el registro durante el diagnóstico y durante los ensayos conductuales de la habilidad en cuestión.

Para los fines de este artículo se mostrarán, a manera de ejemplo, los componentes de tres de las siete habilidades practicadas, dado que las siete habilidades comparten algunas conductas verbales y no verbales, y las experiencias obtenidas fueron similares entre las tres expuestas y las cuatro restantes.

Respecto a la habilidad: Escuchar, que implica tratar de entender las palabras, sentimientos y emociones de los pacientes, así como valorar sus preocupaciones y problemas ${ }^{3,4}$, se muestran en la Tabla 1 , tanto conductas verbales como no verbales que incorporan elementos básicos de clarificación, paráfrasis, reflejo y síntesis propios de la adquisición de la habilidad de Escucha ${ }^{3}$. La mayoría requirió de dos o tres ensayos para el logro de la habilidad, la falla más frecuente en las conductas verbales se centró en el componente refleja los sentimientos y frases del otro y más de una tercera parte no lo logró al terminar el ejercicio de esta habilidad, hecho que motivó una práctica adicional como refuerzo. En las conductas no verbales, también se observó que algunos participantes requirieron de dos ensayos conductuales para el logro de dichas conductas; no obstante, desde el diagnóstico también se advirtió la presencia de varios componentes en la mayoría de las enfermeras como la mirada, gesticulaciones y tono de voz.

Tabla 1. Proporciones respecto al Diagnóstico inicial y Aprendizaje de las enfermeras en la habilidad: Escuchar ( $\mathbf{n = 1 4 )}$

\begin{tabular}{|c|c|c|c|c|c|c|}
\hline \multirow[t]{2}{*}{ Conductas verbales y no verbales } & \multicolumn{2}{|c|}{$\begin{array}{l}\text { Componente en } \\
\text { el Diagnóstico }\end{array}$} & \multicolumn{3}{|c|}{$\begin{array}{c}\text { Ensayo en que se } \\
\text { presenta el Aprendizaje }\end{array}$} & \multirow{2}{*}{$\begin{array}{c}\text { No se } \\
\text { obtuvo el } \\
\text { Aprendizaje }\end{array}$} \\
\hline & Presente & Ausente & Primero & Segundo & Tercero & \\
\hline \multicolumn{7}{|l|}{ Verbales } \\
\hline - Se presenta al paciente diciendo su nombre & .43 & .57 & .62 & .38 & -- & -- \\
\hline $\begin{array}{l}\text { - Le hace preguntas al paciente } \\
\text { para clarificar el mensaje }\end{array}$ & .71 & .29 & 1.00 & -- & -- & -- \\
\hline - Refleja los sentimientos y frases del otro & .14 & .86 & .17 & .33 & .08 & .42 \\
\hline - Resume los mensajes del otro & .79 & .21 & 1.00 & -- & -- & -- \\
\hline - Asiente con monosílabos & .79 & .21 & 1.00 & -- & -- & -- \\
\hline \multicolumn{7}{|l|}{ No verbales } \\
\hline - Mira al otro el $80 \%$ del tiempo aproximadamente & .71 & .29 & .75 & .25 & -- & -- \\
\hline - Le sonríe & .50 & .50 & .43 & .43 & .14 & -- \\
\hline - Inclina ligeramente su cuerpo hacia el otro & .57 & .43 & .83 & .17 & -- & -- \\
\hline - Asiente con la cabeza & .86 & .14 & 1.00 & -- & -- & -- \\
\hline - Manifiesta gestos que muestran atención & .93 & .07 & 1.00 & -- & -- & -- \\
\hline - Utiliza tono de voz audible & .86 & .14 & .50 & .50 & -- & -- \\
\hline - Mantiene una postura relajada ante el paciente & .71 & .29 & .50 & .25 & -- & .25 \\
\hline
\end{tabular}


En el ejercicio de la habilidad: Responder a los sentimientos del otro, que implica una actitud empática para ponerse auténticamente en el lugar del paciente y tratar de ver el mundo como él lo ve ${ }^{3,4}$, con el apoyo de elementos como la paráfrasis y el reflejo de sentimientos ${ }^{3}$. Los participantes mostraron de nuevo deficiencia en el reflejo de sentimiento, pero en casi todos los casos se retomó la conducta en el primer ensayo, la mayoría la llevo a cabo en el primer ensayo y el resto en el segundo. Los componentes no verbales prácticamente estuvieron presentes desde el diagnóstico, como se aprecia en la Tabla 2.

Tabla 2. Proporciones respecto al Diagnóstico inicial y Aprendizaje de las enfermeras en la habilidad: Responder a los sentimientos del otro $(n=14)$

\begin{tabular}{|c|c|c|c|c|c|c|}
\hline \multirow[t]{2}{*}{ Conductas verbales y no verbales } & \multicolumn{2}{|c|}{$\begin{array}{l}\text { Componente en } \\
\text { el Diagnóstico }\end{array}$} & \multicolumn{3}{|c|}{$\begin{array}{l}\text { Ensayo en que se } \\
\text { presenta el Aprendizaje }\end{array}$} & \multirow{2}{*}{$\begin{array}{c}\text { No se } \\
\text { obtuvo el } \\
\text { Aprendizaje }\end{array}$} \\
\hline & Presente & Ausente & Primero & Segundo & Tercero & \\
\hline \multicolumn{7}{|l|}{ Verbales } \\
\hline $\begin{array}{l}\text { - Le dice al otro lo que observa en su rostro } \\
\text { y en su cuerpo (reflejo de sentimientos); } \\
\text { y puede repetir las palabras del otro }\end{array}$ & .36 & .64 & .89 & .11 & -- & -- \\
\hline $\begin{array}{l}\text { - Le expresa al otro con sinceridad y claridad lo } \\
\text { que piensa y siente sobre los sentimientos del otro }\end{array}$ & .57 & .43 & 1.00 & -- & -- & -- \\
\hline \multicolumn{7}{|l|}{ No verbales } \\
\hline $\begin{array}{l}\text { - Observa la expresión y acciones del otro } \\
\text { y lo mira al menos el } 80 \% \text { del tiempo }\end{array}$ & .93 & .07 & 1.00 & -- & -- & -- \\
\hline - Asiente con la cabeza & 1.00 & .00 & -- & -- & -- & -- \\
\hline - Manifiesta gestos que muestran atención & 1.00 & .00 & -- & -- & -- & -- \\
\hline - Utiliza tono de voz audible & 1.00 & .00 & -- & -- & -- & -- \\
\hline $\begin{array}{l}\text { - Mantiene una postura corporal } \\
\text { relajada frente al paciente }\end{array}$ & .93 & .07 & 1.00 & -- & -- & -- \\
\hline
\end{tabular}

Nota. Si el componente estuvo ausente en el Diagnóstico, en las dos siguientes columnas se muestra la proporción que logró el cambio según ensayo, el cual mostró el 100\% de los que carecían de esta habilidad.

Respecto a la habilidad: Responder a una queja, que requiere del entendimiento, por parte de la enfermera, del estado de ansiedad, incertidumbre e intranquilidad que pueden magnificar los acontecimientos observados y experimentados por el paciente. En el diagnóstico se identificaron deficiencias en la mayoría de las componentes verbales, pero se alcanzó la conducta en el primer ensayo y en pocos casos se necesitó de un segundo intento, previa retroalimentación. En los comportamientos no verbales las enfermeras, casi en su totalidad, mostraron su presencia desde el diagnóstico, sin embargo, el retroceso en algunas conductas previamente adquiridas en la práctica de otras habilidades pudo haberse relacionado con la condición establecida para el aprendizaje de esta habilidad que suponía una actitud negativa del paciente, situación que no estaba presente en la práctica de las habilidades ejercitadas con anterioridad como: Expresar aliento, Expresar un cumplido y Responder a los sentimientos del otro (Tabla 3).

En la última sesión del entrenamiento, se les pidió que de forma voluntaria expresaran verbalmente o por escrito el resultado de la vivencia personal derivada del aprendizaje logrado durante la práctica del programa, así como considerar el ejercicio profesional y su experiencia personal con familiares, compañeros y amigos. Entre los comentarios expresados destacan:

...no sé por qué cada año nos actualizan en arritmias y no en aspectos humanos con el paciente, nos hace mucha falta...

...nos ayudó a entender mejor al paciente, pero me cuesta mucho cuando están deprimidos o enojados...

...me aportó en lo profesional y en lo personal, me ayuda al objetivo del cuidado y mejoría del paciente...

....me cuesta escuchar, me ayudó a reflexionar...

... aunque tenemos mucho trabajo, podré aprovechar...

...me es difícil dar información negativa al paciente, aprendí alguna forma...

....me cuesta decirles que ya están fuera de tratamiento quirúrgico, pero entendí que puedo apoyar un poco...

...no solo mejoré la comunicación con el paciente, también con mi entorno... 
Tabla 3. Proporciones respecto al Diagnóstico inicial y Aprendizaje de las enfermeras en la habilidad: Responder a una queja. $(n=14)$

\begin{tabular}{|c|c|c|c|c|c|c|}
\hline \multirow[t]{2}{*}{ Conductas verbales y no verbales } & \multicolumn{2}{|c|}{$\begin{array}{l}\text { Componente en } \\
\text { el Diagnóstico }\end{array}$} & \multicolumn{3}{|c|}{$\begin{array}{c}\text { Ensayo en que se presenta } \\
\text { el Aprendizaje }\end{array}$} & \multirow{2}{*}{$\begin{array}{c}\text { No se } \\
\text { obtuvo el } \\
\text { Aprendizaje }\end{array}$} \\
\hline & Presente & Ausente & Primero & Segundo & Tercero & \\
\hline \multicolumn{7}{|l|}{ Verbales } \\
\hline $\begin{array}{l}\text { - Le hace preguntas para comprender } \\
\text { el sentido de la queja expresada }\end{array}$ & .64 & .36 & .80 & .20 & -- & -- \\
\hline - Le menciona que comprende las razones & .57 & .43 & 1.00 & -- & -- & -- \\
\hline $\begin{array}{l}\text { - Expresa lo que siente y lo que piensa, } \\
\text { acepta su responsabilidad si es el caso }\end{array}$ & .71 & .29 & .75 & .25 & -- & -- \\
\hline $\begin{array}{l}\text { - Resume con el otro los pasos que } \\
\text { se siguieron para exponer su queja y } \\
\text { su responsabilidad si es el caso }\end{array}$ & .36 & .64 & .89 & .11 & & \\
\hline \multicolumn{7}{|l|}{ No verbales } \\
\hline - Mira al otro el 80\% del tiempo apróx. & 1.00 & .00 & -- & -- & -- & -- \\
\hline - Le permite expresar la queja, lo escucha & .93 & .07 & -- & 1.00 & -- & -- \\
\hline - Asiente con la cabeza & 1.00 & .00 & -- & -- & -- & -- \\
\hline - Manifiesta gestos que muestran atención & 1.00 & .00 & -- & -- & -- & -- \\
\hline $\begin{array}{l}\text { - Se aproxima físicamente al otro, } \\
\text { fijándose si permite el acercamiento }\end{array}$ & 1.00 & .00 & -- & -- & -- & -- \\
\hline - Le sonríe & .71 & .29 & 1.00 & -- & -- & -- \\
\hline - Utiliza tono de voz audible y firme & 1.00 & .00 & -- & -- & -- & -- \\
\hline - Inclina ligeramente su cuerpo hacia el otro & .79 & .21 & 1.00 & -- & -- & -- \\
\hline
\end{tabular}

Nota. Si el componente estuvo ausente en el Diagnóstico, en las dos siguientes columnas se muestra la proporción que logró el cambio según ensayo, por lo tanto mostro el 100\% de los que carecían de esta habilidad.

\section{Conclusiones}

Los datos plasmados en las tablas, permiten identificar que casi todas las enfermeras ya contaban con varios de los componentes de las diferentes habilidades, aunque, la realización del programa de entrenamiento les dio la oportunidad de complementar conductas que no estaban presentes en el diagnóstico, sobre todo las de tipo verbal específicas de cada habilidad y algunas de tipo no verbal que rápidamente fueron asimiladas durante el desarrollo del programa, como la sonrisa, la posición corporal y el contacto físico. Cabe resaltar que las estrategias cognitivo conductuales permiten que el aprendiz experimente la carencia de una conducta y pueda reforzarla a partir de la retroalimentación de los facilitadores del programa de entrenamiento.

Los comentarios adicionales ofrecen información que puede sustentar la necesidad sentida y el interés para implementar programas similares que benefician tanto a los pacientes como a las enfermeras, y que pueden repercutir en las evaluaciones de calidad de los servicios en el rubro de satisfacción del cuidado recibido en cuanto a la interacción personal.

\section{Responsabilidades éticas}

Protección de personas y animales. Las autoras declaran que para esta investigación no se han realizado experimentos en seres humanos o animales.

Confidencialidad de los datos. Las autoras declaran que han seguido los protocolos de su centro de trabajo sobre la publicación de datos de pacientes.

Financiamiento. Ninguno.

Conflicto de intereses. Ninguno 


\section{Referencias}

1. Arnold EC, Underman-Boggs K. Interpersonal Relationships. Professional Communication Skills for Nurses. $6^{\text {th }}$ ed.EE.UU: Elsevier Saunders; 2011.

2. Tazón-Ansola P, Aseguinolaza-Chopitea L, García-Campayo J. Ciencias Psicosociales. Barcelona: Masson; 2000.

3. CormierWH, Cormier LS. Estrategias de entrevista para terapeutas. Habilidades básicas e intervenciones cognitivo-conductuales. $3^{\text {ra }}$ ed. Bilbao: Descleé de Brouwer; 2000.

4. Cibanal-Juan L, Arce-Sánchez MC, Carballal-Balsa MC. Técnicas de comunicación y relación de ayuda en ciencias de la salud. $2^{\text {da }}$ ed. Barcelona: Elsevier; 2010.

5. Watson J. Assessing and Measuring Caring in Nursing and Health Sciences. $2^{\text {nd }}$ ed. New York: Springer Publishing Company; 2009.

6. McGilton K, Irwin-Robinson H, Boscard V, Spanjevic L. Communication enhancement: nurse and patient satisfaction outcomes in a complex continuing care facility. J Adv Nurs. 2006; 54(1): 35-44. https://doi.org/10.1111/j.1365-2648.2006.03787.x

7. Ranmal R, Prictor M, Scott JT. Interventions for improving communication with children and adolescents about their cancer. Cochrane Database Syst Rev. 2008; 8(4): CD002969.

https://doi.org/10.1002/14651858.CD002969.pub2

8. Uitterhoeve RJ, Bensing JM, Grol RP, Demulder PHM, Van Achterberg T. The effect of communication skills training on patient outcomes in cancer care: a systematic review of the literature. Eur J Cancer Care. 2010; 19(4): 442-57. https://doi.org/10.1111/j.1365-2354.2009.01082.x

9. Barth J, Lannen P. Efficacy of communication skills training courses in oncology: a systematic review and meta-analysis. Ann Oncol. 2011; 22(5): 1030-40. https://doi.org/10.1093/annonc/mdq441

10. Doyle D, Copeland HL, Bush D, Stein L, Thompson S. A course of nurses to handle difficult communication situations. A randomized controlled trial of impact on self-efficacy and performance. Patient Educ Couns. 2011; 82(1): 100-9. https://doi.org/10.1016/j.pec.2010.02.013

11. Müggenburg C, Riveros-Rosas A, Juárez-García F. Entrenamiento en habilidades de comunicación en enfermeras y percepción del paciente que recibe su cuidado. Enferm univ. 2016; 13(4): 201-7. https://doi.org/10.22201/eneo.23958421e.2016.4.65

12. Goldstein AP, Soprafkin RP, Gershaw NJ. Sé lo que anda mal, pero... no sé qué hacer. México: Diana; 1993.

13. Roth-Unzueta E. Competencia Social: el cambio del comportamiento individual en la comunidad. Ciudad de México: Trillas; 1986. 